\title{
Reconstruction of a Severely Crushed Leg with Interpositional Vessel Grafts and Latissimus Dorsi Flap
}

\author{
Chan Woo Park ${ }^{1}$, Youn Hwan Kim ${ }^{1}$, Kyu Tae Hwang ${ }^{2}$, Jeong Tae Kim ${ }^{1}$ \\ ${ }^{1}$ Department of Plastic and Reconstructive Surgery, ${ }^{2}$ Department of Orthopeadic Surgery, Hanyang University School of Medicine, Seoul, \\ Korea
}

We present a case of a near total amputation at the distal tibial level, in which the patient emphatically wanted to save the leg. The anterior and posterior tibial nerves were intact, indicating a high possibility of sensory recovery after revascularization. The patient had open fractures at the tibia and fibula, but no bone shortening was performed. The posterior tibial vessels were reconstructed with an interposition saphenous vein graft from the contralateral side and a usable anterior tibial artery graft from the undamaged ipsilateral distal portions. The skin and soft tissue defects were covered using a subatmospheric pressure system for demarcating the wound, and a latissimus dorsi myocutaneous free flap for definite coverage of the wound. At 6 months after surgery, the patient was ambulatory without requiring additional procedures. Replantation without bone shortening, with use of vessel grafts and temporary coverage of the wound with subatmospheric pressure dressings before definite coverage, can shorten recovery time.

Keywords Limb salvage / Negative-pressure wound therapy / Vascular grafting
Correspondence: Youn Hwan Kim Department of Plastic and Reconstructive Surgery, Hanyang University School of Medicine, 222

Wangsimni-ro, Seongdong-gu, Seoul 133-791, Korea

Tel: $+82-2-2290-8560$

Fax: +82-2-2295-7671

E-mail:younhwank@daum.net

\section{INTRODUCTION}

The efficacy of replantation of the lower extremities is controversial. Crushing injuries, which render replantation technically more difficult, are a common cause of lower extremity amputations. In unilateral amputations, stump revision and applying prostheses can provide a limb that is equally functional to the original, and costs less than replantation [1-3]. For these reasons, there have been fewer reports of lower extremity replantation than that of upper extremity replantation [4-8].

The authors report on a case of replantation of a unilateral limb that was severely crushed and nearly amputated at the distal tib- ial level, without any limb shortening, which allowed the patient to regain full ambulation 6 months after surgery.

\section{CASE}

A 29-year-old male was transported to the emergency department after a traumatic near total amputation of the right distal tibia due to a motorcycle accident; the patient's leg was run over by a truck. The distal portion of the leg was severely crushed and the only remaining portions were the anterior and posterior tibial nerves, the tibialis anterior tendon, and some soft tissue connections, with no viable blood flow to the distal stump (Fig.

Copyright $(\odot 2012$ The Korean Society of Plastic and Reconstructive Surgeons

This is an Open Access article distributed under the terms of the Creative Commons Attribution Non-Commercial License (http://creativecommons.org/

licenses/by-nc/3.0/) which permits unrestricted non-commercial use, distribution, and reproduction in any medium, provided the original work is properly cited.

www.e-aps.org 


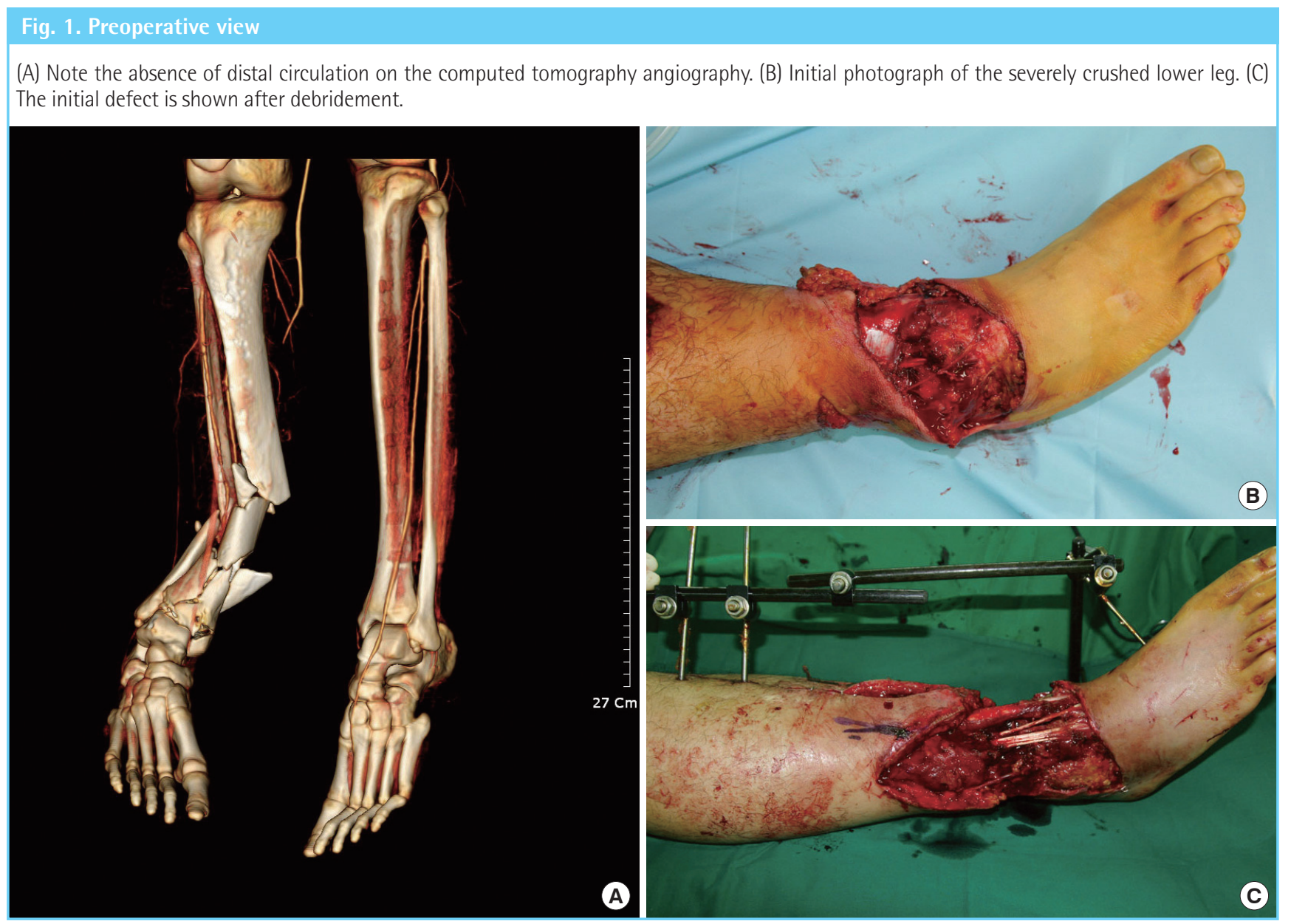

1A). The amputation site was critically contaminated, with circumferential degloving tissue loss of approximately $15 \mathrm{~cm}$ in length at the distal portion of the lower leg (Fig. 1B).

A comminuted fracture of the tibia and fibula was observed at the distal portions with damage to the ankle joint. Despite the severity of the damage to the amputated region, the general condition of the patient remained fairly stable. The total ischemia time was more than 10 hours of warm ischemia.

After an external fixator was applied to the open tibial fracture and internal fixation was performed for the fibula, wound exploration was performed (Fig. 1C). Thrombosis of the anterior tibial artery and a 20-cm-long segment of the artery's crushed intimal structure were observed, along with a similarly damaged vein. The posterior tibial vessels showed an arterial gap of $10 \mathrm{~cm}$, with one accompanying vein ruptured and the other vein crushed with a venous gap of $7 \mathrm{~cm}$ (Fig. 2A). The posterior tibial vessels appeared more promising for a successful repair. A lesser saphenous vein graft was harvested from the contralateral leg in a $12 \mathrm{~cm}$ segment for the arterial reconstruction (Fig. 2B). After blood flow resumed, $400 \mathrm{~mL}$ of blood was drained before the vein was repaired.
The ruptured vein was repaired primarily, and the vein with a $7 \mathrm{~cm}$ gap was repaired with an $8 \mathrm{~cm}$ strip of arterial graft from a non-thrombosed segment at the middle portion of the thrombosed anterior tibial artery (Fig. 2).

The healthy vein graft was considered more reliable for the arterial reconstruction than the arterial graft from the damaged anterior tibial artery. Therefore, the posterior tibial arterial reconstruction was achieved using the $12 \mathrm{~cm}$ harvested contralateral lesser saphenous vein graft. The skin and soft tissue defect around the proximal stump underwent minimal debridement and the Vacuum-Assisted Closure System (KCI Medical, San Antonio, TX, USA) was applied for demarcating the necrotizing portions. To protect the anastomosis site, vessel graft site coverage was performed using overlying soft tissue remnants and a bovine dermal substitute (Matriderm, Dr. Suwelak Skin and Health Care, Billerbeck, Germany), while avoiding applied direct negative suction pressure. During the operation, $800 \mathrm{~mL}$ of whole blood was transfused. Prostaglandin E1 (Eglandin, Mitsubishi Tanabe Pharma Korea, Seoul, Korea) and heparin were administered for 2 weeks postoperatively. After the replantation, there were no immediate complications such as pulmonary 


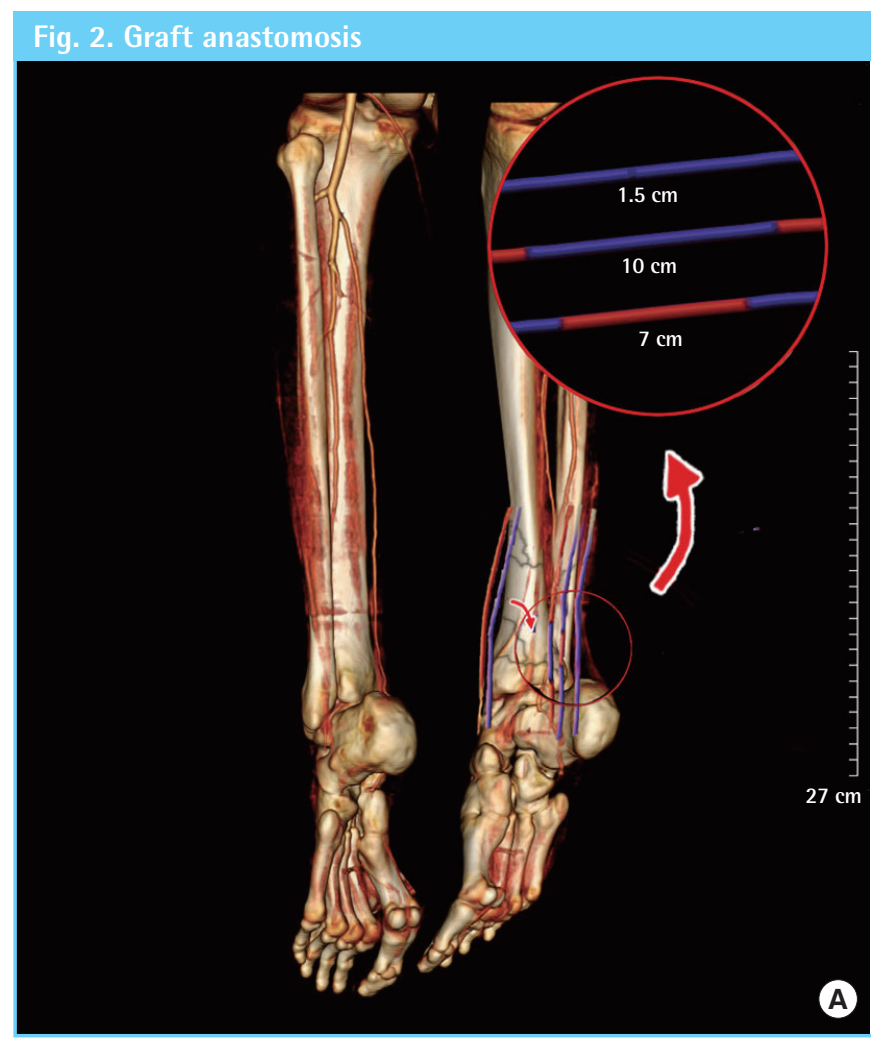

Fig. 3. Intraoperative view

Latissimus dorsi myocutaneous flap.

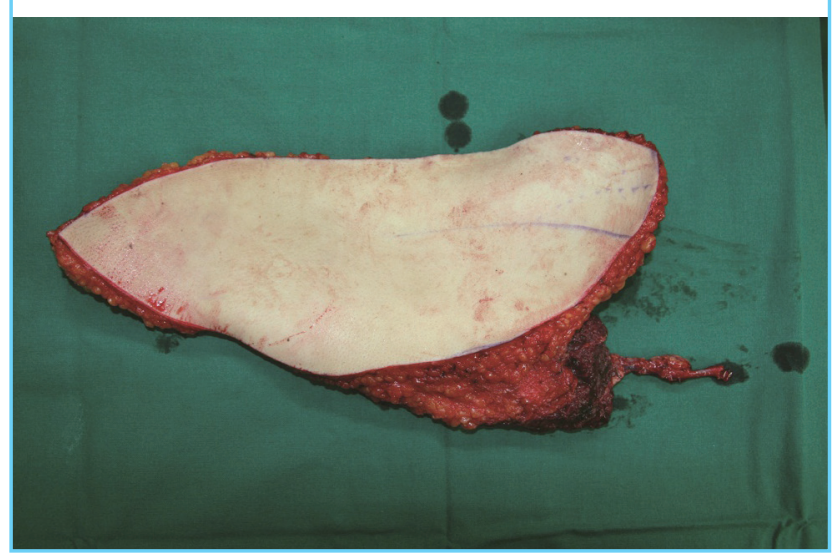

thromboembolism or shock. Two weeks after the replantation, the wound was demarcated and clean. No definite sign of infection was observed.

Internal fixation of the tibial bone was carried out and coverage of the defect with a $25 \times 15 \mathrm{~cm}$ sized latissimus dorsi myocutaneous flap was performed (Fig. 3). The thoracodorsal artery and vein were anastomosed to the middle $1 / 3$ anterior tibial vessels, where undamaged structures in an end-to-end fashion were found. Split-thickness skin grafts were performed simultaneously to cover portions without exposing major structures. The flap healed well, but long-standing use of a splint resulted in
(A) A schematic drawing of the reconstruction of the posterior tibial vessels. (B) After repair of the posterior tibial vessels. An arterial graft from the non-traumatized portions of the ipsilateral anterior tibial artery and a saphenous vein graft from the contralateral side were used to bridge the defect. A black arrow indicates the reconstructed posterior tibial vein with the non-thrombosed segment of the ipsilateral anterior tibial artery. A white arrow indicates the reconstructed posterior tibial artery with the contralateral lesser saphenous vein.

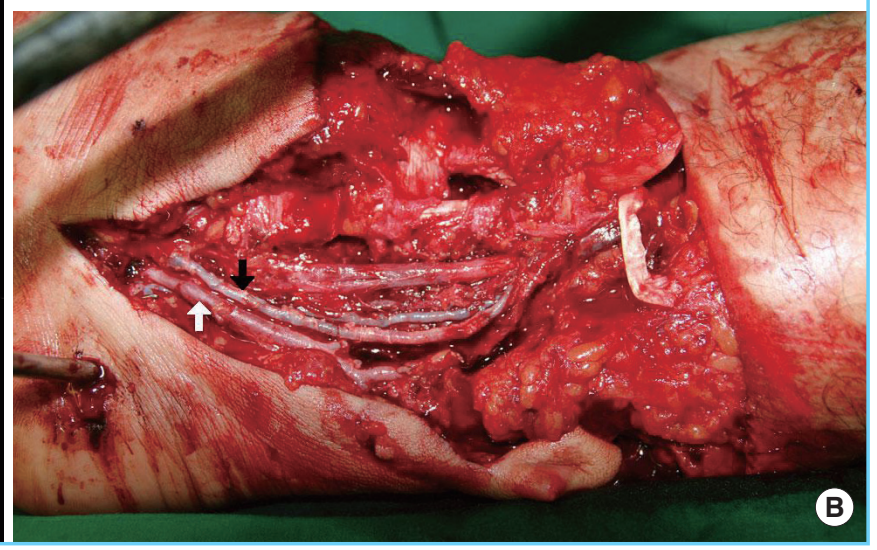

a pressure sore at the heel region with exposure of the Achilles tendon.

We performed coverage with bovine dermal substitute (Matriderm, Dr. Suwelak Skin and Health Care, Germany) and splitthickness skin graft to the area 3 weeks after the second surgery.

The patient was discharged soon afterwards, and was walking with crutches (Fig. 4A). Six months after the operation, the patient was ambulatory without aid, and no additional procedures were required (Fig. 4B). Twelve months after the operation, sensory recovery of the foot sole was quantitatively measured using two-point discrimination sensation by a esthesiometer. The two-point discrimination sensation on the damaged foot sole was measured at $14 \mathrm{~mm}$ and that of the patient's healthy foot sole was measured at $13 \mathrm{~mm}$. The sensation of the damaged foot sole was not significantly different from that of the healthy foot sole. A grade II in the Chen criteria of the replanted lower limb was achieved. The patient and his family were satisfied with the results.

\section{DISCUSSION}

Lower limb replantation presents many challenges to a reconstructive surgeon even though microsurgery has evolved remarkably in recent years. Severe crush wounds require numerous surgical procedures with prolonged rehabilitation; other life threatening injuries accompanied with heavy exsanguination of- 


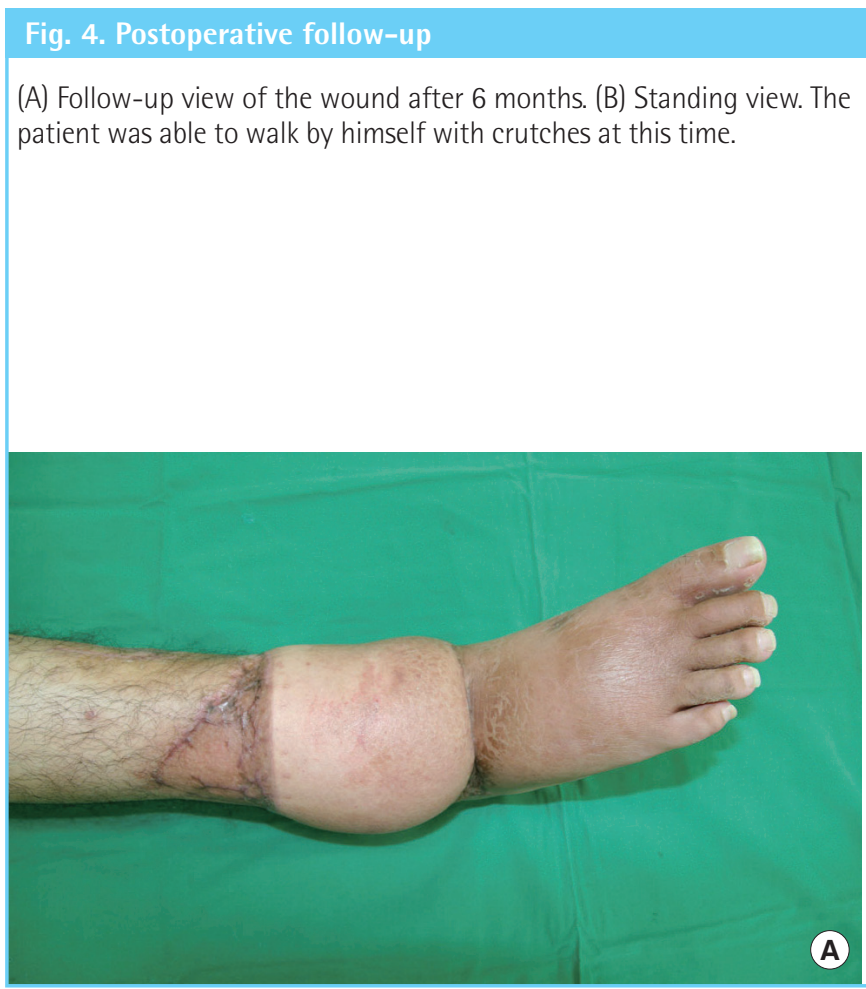

ten lead to amputation rather than replantation $[1,2]$. Moreover, advanced artificial lower limbs can provide functions similar to that of a replanted foot, at a much reduced cost $[2,3,5]$.

The indication of lower limb replantation is still controversial $[1,2,4,6,7]$. For infants, an amputated body part is considered to be a candidate for replantation, and in bilateral amputations for infants, replantation of at least one extremity is indicated, even in a heterotopic form $[2,4,7]$. Helfet et al. [9] composed a scoring system for severely damaged lower extremities with vascular compromise that integrates the patient's age, presence of shock, ischemic time, and skeletal or soft-tissue injuries. Battiston et al. [2] further advanced the scoring system exclusively for amputations, and included the importance of nerve damage into the score.

According to both scoring systems, our patient was contraindicated for replantation, showing a Mangled Extremity Severity Score score of 10 according to the Helfet system and a score of 9 according to the Battiston system, which are higher than the cutoff values of 7 and 8 for the two scoring systems, respectively. However, because the patient and his parents had emphatically wanted the replantation, we underwent the surgery, accepting the risks. A point worth mentioning is that in Asian countries, many retain a cultural practice of taking off one's shoes when going indoors, which might allow a broader indication for replantation for patients within the cultural expectation of revealing one's feet a wider range of circumstances [6].

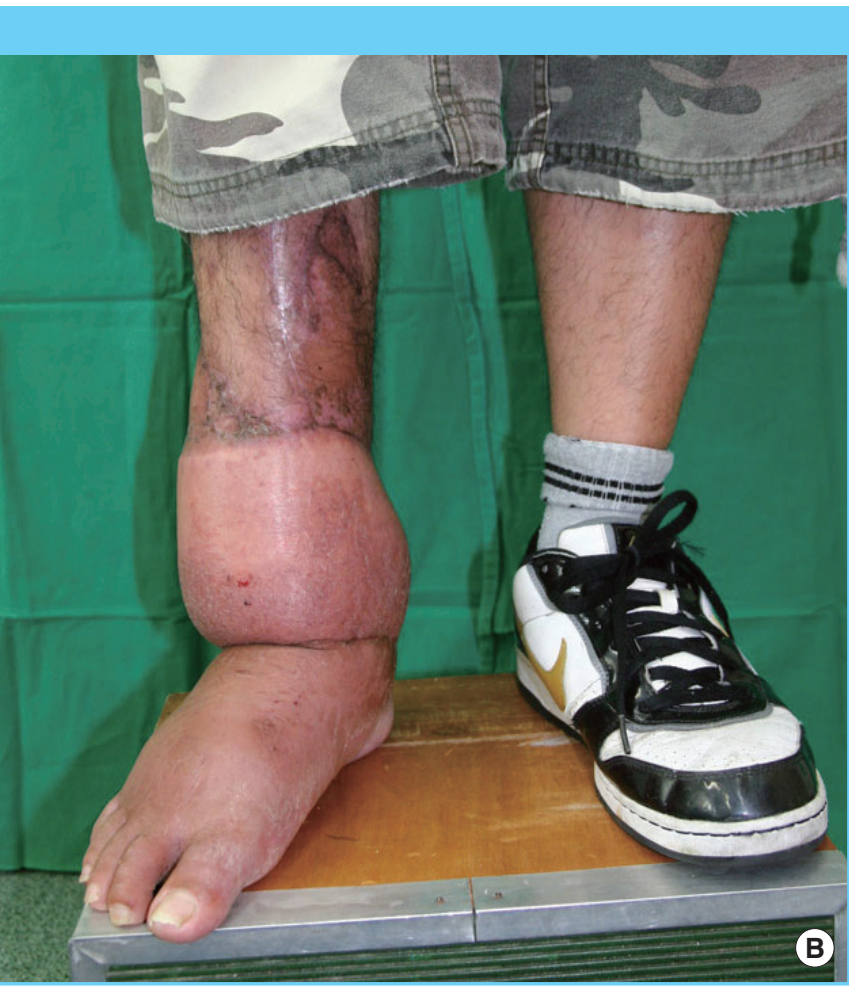

Hierner et al. [1] presented 4 adjunct criteria as preconditions for replantation, which are as follows: perfect viability of the replanted part, lack of severe systemic disturbance due to the replantation, a functional extremity, and an acceptable length of time for rehabilitation with the ability to return to normal life. The criterion of a functional extremity is a sensate sole, which renders the replanted part different from prostheses $[2,4]$. In our case, the anterior and posterior tibial nerves were intact, which influenced our choice to proceed with the replantation.

Many reports have presented limb shortening as necessary for achieving complete debridement and closure of soft tissues, direct coaptation of severed nerves, and direct anastomosis of cut vessels $[1,2,4,5]$. These procedures are followed by limb lengthening procedures with an llizarov apparatus, which aids the bone lengthening over a $1-2$ month period $[1,2,4,5]$. In this case, no shortening was performed even though there were significant defects of the vessels and soft tissue combined with a severely comminuted fracture. The vessel gaps were bridged with the liberal use of vessel grafts.

We utilized an arterial graft from the zone of injury with intact intimal structures based upon microscopic examination. Our concern over further necrosis of the damaged tissue compelled us to delay the closure of the wound. Vacuum-assisted closure was used for 2 weeks before coverage of the wound with a latissimus dorsi myocutaneous free flap was performed. Even though flap coverage during the first week of treatment has shown 
decreased complication rates such as flap failure and infection, Rinker et al. [10] showed that using a subatmospheric dressing could bring down the complication rates of flap coverage within 6 weeks similarly to that performed in the acute stage. Following this procedure allowed us more time to observe the wound for any further necroses that would have necessitated debridement.

\section{REFERENCES}

1. Hierner R, Betz AM, Comtet JJ, et al. Decision making and results in subtotal and total lower leg amputations: reconstruction versus amputation. Microsurgery 1995;16:830-9.

2. Battiston B, Tos P, Pontini I, et al. Lower limb replantations: indications and a new scoring system. Microsurgery 2002;22:187-92.

3. Busse JW, Jacobs CL, Swiontkowski MF, et al. Complex limb salvage or early amputation for severe lower-limb injury: a meta-analysis of observational studies. J Orthop Trauma 2007;21:70-6.

4. Cavadas PC, Landin L, Ibanez J, et al. Infrapopliteal lower extremity replantation. Plast Reconstr Surg 2009; 124:532-9.

5. Nisanci M, Er E, Yildiz C, et al. Replantation of a crush am- putation of distal tibia followed by lengthening with Ilizarov circular external fixator: two-year follow-up. Microsurgery 2002;22:295-9.

6. Nagamatsu S, Sakayama K, Kamogawa J, et al. Replantation of severed foot at the chopart joint: a case report. Foot Ankle Int 2009;30:1229-32.

7. Kayikcioglu A, Agaoglu G, Nasir S, et al. Crossover replantation and fillet flap coverage of the stump after ectopic implantation: a case of bilateral leg amputation. Plast Reconstr Surg 2000;106:868-73.

8. Lew DH, Yun JY, Tark KC, et al. Lower leg salvage procedure in massive bone \& soft tissue defects: combined free flap \& ilizarov distraction osteogenesis. J Korean Soc Plast Reconstr Surg 1999;26:938-44.

9. Helfet DL, Howey T, Sanders R, et al. Limb salvage versus amputation. Preliminary results of the Mangled Extremity Severity Score. Clin Orthop Relat Res 1990;(256):80-6.

10. Rinker B, Amspacher JC, Wilson PC, et al. Subatmospheric pressure dressing as a bridge to free tissue transfer in the treatment of open tibia fractures. Plast Reconstr Surg 2008; 121:1664-73. 